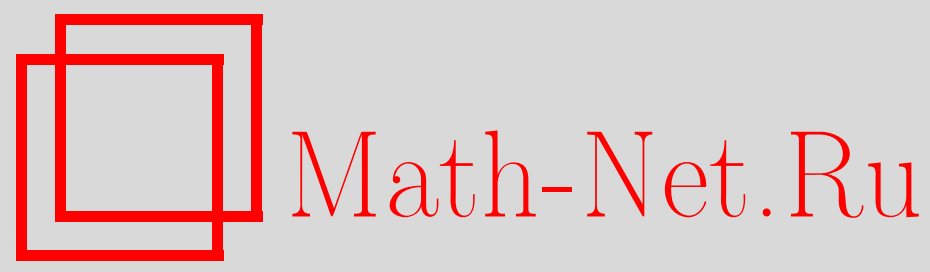

М. Р. Алеева, О конечных простых группах с множеством порядков элементов, как у группы Фробениуса или двойной группы Фробениуса, Матем. заметки, 2003, том 73, выпуск 3, 323-339

DOI: https://doi.org/10.4213/mzm189

Использование Общероссийского математического портала Math-Net.Ru подразумевает, что вы прочитали и согласны с пользовательским соглашением http://www.mathnet.ru/rus/agreement

Параметры загрузки:

IP : 3.85 .5 .30

26 апреля 2023 г., 12:54:24

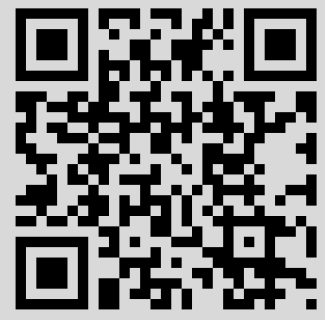




\title{
О КОНЕЧНЫХ ПРОСТЫХ ГРУППАХ \\ С МНОЖЕСТВОМ ПОРЯДКОВ ЭЛЕМЕНТОВ, КАК У ГРУППЫ ФРОБЕНИУСА ИЛИ ДВОЙНОЙ ГРУППЫ ФРОБЕНИУСА
}

\author{
М.Р. Алеева
}

Доказано, что конечная простая группа с множеством порядков элементов как у группы Фробениуса (соответственно двойной группы Фробениуса) изоморфна $L_{3}(3)$ или $U_{3}(3)$ (соответственно $U_{3}(3)$ или $\left.S_{4}(3)\right)$.

Библиография: 24 названия.

1. Введение. Пусть $G$ - конечная группа. Обозначим через $\omega(G)$ множество всех порядков элементов групш $G$. Это множество частично упорядочено относительно делимости и потому однозначно определяется подмножеством $\mu(G)$ своих максимальных по делимости элементов. Для натурального числа $n$ через $\pi(n)$ обозначим множество всех простых делителей числа $n$ и положим $\pi(G)=\pi(|G|)$. Множество $\omega(G)$ определяет граф Грюнберга-Кегеля $G K(G)$, или граф простых чиселгруппы $G$, множеством вершин которого служит $\pi(G)$, и две вершины $p, q$ из $\pi(G)$ соединены ребром, если $G$ содержит элемент порядка $p q$. Обозначим через $\pi_{i}=\pi_{i}(G)$, где $i=1, \ldots, t(G), i$-ю связную компоненту графа $G K(G)$. Для группы $G$ четного порядка положим $2 \in \pi_{1}$. Обозначим через $\mu_{i}=\mu_{i}(G)$ множество тех $n \in \mu(G)$, для которых $\pi(n) \subseteq \pi_{i}$.

Грюнберг и Кегель доказали следующую структурную теорему для конечных груш с несвязным графом простых чисел.

ТЕОРемА ГРЮНБЕРГА-КЕГЕЛЯ (см. [1, теорема А]). Ecли $G$ - конечная группа $c$ несвязным графом Грюнберга-Кегеля, то верно одно из следующих утверэсдений:

(a) $G$ - әруппа Фробениуса;

(б) $G=A B C$, где $A, A B$ - нормальные подгруппь группь $G$ и $A B, B C-$ группь Фробениуса с ядрами $A, B$ и дополнениями $B, C$ соответственно;

(в) $G$ является расширением $\pi_{1}(G)$-группы $N$ посредством группы $A$, где $P \leqslant$ $A \leqslant \operatorname{Aut}(P), P$ - простая неабелева группа с несвязным графом $G K(P)$, $A / P-\pi_{1}(G)$-2pynna.

Естественно возникает

Работа выполнена при финансовой поддержке Российского фонда фундаментальных исследований, грант № 02-01-00781. 
Вопрос. Какие конечные простыегрупшы имеют то же множество порядков элементов, как группы из пунктов (а) или (б) теоремы Грюнберга-Кегеля?

Используя классификацию конечных простых групп с несвязным графом Грюнберга-Кегеля (см. [1]-[3]), мы доказываем следующую теорему.

TЕоремА. Пусть $G$ - конечная группа, $\omega(G)=\omega(S)$, где $S$ - конечная простая неабелева группа. Тогда

1) если для группы $G$ выполняется случай (а) теоремы Грюнберга-Кегеля, то $S$ изоморфна $L_{3}(3)$ или $U_{3}(3)$;

2) если для группы $G$ выполняется случай (б) теоремы Грюнберга-Кегеля, то $S$ изоморфна $U_{3}(3)$ или $S_{4}(3)$.

Если группа $S$ изоморфна $L_{3}(3)$ или $U_{3}(3)$, то построены примеры групп Фробениуса $G$ таких, что $\omega(G)=\omega(S)$ (см. примеры 1 и 2). Вопрос о существовании двойных групा $\Phi$ робениуса $G$ с $\omega(G)=\omega(S)$, где $S$ изоморфна $U_{3}(3)$ или $S_{4}(3)$, остается открытым.

Основные определения и обозначения, принятые в работе, стандартны; их можно найти в [4] или [5].

Везде далее $q$ обозначает некоторую степень простого числа $p$, а $p^{\prime}$ - некоторое нечетное простое число.

Пусть $\delta \in\{-1,1\}$. Положим

$$
S L_{3}^{\delta}(q)=\left\{\begin{array}{ll}
S L_{3}(q), & \text { если } \delta=1, \\
S U_{3}(q), & \text { если } \delta=-1,
\end{array} \quad L_{3}^{\delta}(q)= \begin{cases}L_{3}(q), & \text { если } \delta=1, \\
U_{3}(q), & \text { если } \delta=-1 .\end{cases}\right.
$$

\section{2. Предварительные результаты.}

Лемма 1 (см. [6]-[8]). Пусть $G$ - әруппа Фробениуса с ядром $N$ и дополнением $Н$. Тогда верны следующие утверәсдения:

(1) $N$ - нильпотентная группа; в частности, $|\mu(N)|=1 u G K(N)-$ полный граф;

(2) если $U$ - подгруппа порядка rs из $H$, где $r$ us - простые числа (не обязательно различные), то $U$ - ииклическая группа; в частности, силовские $r$-подгруппы группы $H$ для нечетных простых чисел $r$ ииклические;

(3) если порядок группы $H$ четен, то в $H$ есть единственный әлемент $z$ порядка $2 ;$ в частности, силовская 2-подгруппа группы $H$ является ииклической группой или (обобщенной) группой кватернионов, подгруппа $N$ коммутативна и $n^{z}=n^{-1}$ для любого әлемента $n$ из $\mathrm{N}$;

(4) если $H$ содержит әлемент порядка 16, то H имеет нормальное 2-дополнение;

(5) либо группа $H$ разрешима и граф $G K(H)$ полный, либо $H$ содержит нормальную подгруппу $L \cong S L_{2}(5)$ такую, что $(|L|,|H: L|) \leqslant 2$ и $G K(H)$ мохет быть получен из полного графа на $\pi(H)$ удалением ребра $\{3,5\}$.

ЛЕмма 2 (см. [9, лемма 3]). В случае (б) теоремы Грюнберга-Кегеля $B$ является ииклической группой нечетного порядка, $C$ - ииклическая группа и $G$ содержит элемент порядка

$$
|C| \cdot \prod_{r \in \pi(A)} r
$$


В частности, $G K(B), G K(A C)$ - связные компоненты графа $G K(G)$ и обе являются полными графами.

Лемма 3 (см. [3, лемма 4]). Пусть $H$ - конечная простая группа с $t(H)=2$. Тогда $\left|\mu_{2}(H)\right|=1$. Пусть $n_{2}$ означает единственный әлемент из $\mu_{2}(H)$. Тогда $H, \pi_{1}(H), n_{2}$ таковы, как показано в табличе 1.

Лемма 4. Пусть $G$ - конечная группа $c \omega(G)=\omega(S)$, әде $S$ изоморфна одной из следующих групп: $S_{4}\left(3^{f}\right), f>1, L_{3}^{\delta}(q)$ для четного $q, L_{4}(3),{ }^{2} F_{4}(2)^{\prime},{ }^{3} D_{4}(2), S_{6}(2)$, $\mathrm{O}_{8}^{+}(2), M_{12}, \mathrm{Ru}, \mathrm{He}, \mathrm{McL}, \mathrm{Co}_{1}, \mathrm{Co}_{3}, \mathrm{Fi}_{22}, \mathrm{~F}_{5}, A_{n}$, әде $6<n=p^{\prime}, p^{\prime}+1, p^{\prime}+2$. Тогда $G$ не является группой Фробениуса или двойной группой Фробениуса.

ДокАЗАТЕльСтво. Если $S \cong S_{4}\left(3^{f}\right), f>1$, то см. лемму 13 в [9]. Если $S \cong L_{3}^{\delta}(q)$, $q$ четно, то см. лемму 9 в [10]. Если $S$ изоморфна одной из групा $L_{4}(3),{ }^{2} F_{4}(2)^{\prime},{ }^{3} D_{4}(2)$, $S_{6}(2), O_{8}^{+}(2)$, то см. теорему 2 в [11], предложение 5 в [9] и [12]. Если $S-$ спорадическая группа, то см. [13]-[15]. Если $S \cong A_{n}, 6<n=p^{\prime}, p^{\prime}+1, p^{\prime}+2$, то см. лемму 3 в [3] и лемму 8 в [16].

Лемма 5. Пусть $\omega(G)=\omega\left(L_{3}^{\delta}(q)\right)$, әде q нечетно. Тогда

$$
\begin{array}{ll}
\omega(G)=\left\{p(q-\delta), q^{2}-1, q^{2}+\delta q+1\right\} & \text { npu }(3, q-\delta)=1, \\
\omega(G)=\left\{q-\delta, \frac{p(q-\delta)}{3}, \frac{q^{2}-1}{3}, \frac{q^{2}+\delta q+1}{3}\right\} & \text { npu }(3, q-\delta)=3 .
\end{array}
$$

ДокАЗАТЕЛЬСТво аналогично доказательству леммы 9 в [17] и использует матричные представления элементов группы $S L_{3}^{\delta}(q)$ из [18].

ЛЕмма 6 (теорема Жигмонди, см. [19]). Пусть $p$ - простое число, $n$ - натуральное число, $n \geqslant 2$. Тогда верно одно из следующих утверждений:

(а) существует простое число $s$ такое, что $s$ делит $p^{n}-1$, но $s$ не делит $p^{t}-1$ для всех натуральных чисел $t<n$

(б) $n=6 u p=2$;

(в) $n=2$ и $p=2^{l}-1$ для некоторого простого числа $l$.

Простое число $s$ со свойством (а) называется примитивным простым делителем числа $p^{n}-1$.

Лемма 7 (см. [2, лемма 3а)]). Пусть $a, m$ u $n$ - натуральные числа. Тогда

$$
\left(a^{n}-1, a^{m}-1\right)=a^{(m, n)}-1 .
$$

Лемма 8. Пусть $p$ и $r$ - простые числа $u p^{2}+1=2 r$. Тогда

(а) если $p=2^{l}-1$, где $l$ - простое число, то $p=3, r=5$;

(б) если $p=2^{2^{n}}+1$, где $n \geqslant 0$, то либо $p=3, r=5$, либо $p=5, r=13$. 
ТАБлИцА 1. Конечные простые групшы $H$ с $t(H)=2$

\begin{tabular}{|c|c|c|c|}
\hline$H$ & Ограничения на $H$ & $\pi_{1}(H)$ & $n_{2}$ \\
\hline$\overline{A_{n}}$ & $\begin{array}{c}6<n=p^{\prime}, p^{\prime}+1, p^{\prime}+2 ; \\
\text { одно из } n, n-2 \text { не простое }\end{array}$ & $\overline{\bar{\pi} \pi((n-3) !)}$ & 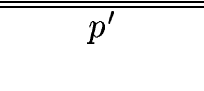 \\
\hline$A_{p^{\prime}-1}(q)$ & $\left(p^{\prime}, q\right) \neq(3,2),(3,4)$ & $\pi\left(q \prod_{i=1}^{p^{\prime}-1}\left(q^{i}-1\right)\right)$ & $\frac{q^{p^{\prime}}-1}{(q-1)\left(p^{\prime}, q-1\right)}$ \\
\hline$A_{p^{\prime}}(q)$ & $(q-1) \mid\left(p^{\prime}+1\right)$ & $\pi\left(q\left(q^{p^{\prime}+1}-1\right) \prod_{i=1}^{p^{\prime}-1}\left(q^{i}-1\right)\right)$ & $\frac{q^{p}-1}{q-1}$ \\
\hline${ }^{2} A_{p^{\prime}-1}(q)$ & & $\pi\left(q \prod_{i=1}^{p^{\prime}-1}\left(q^{i}-(-1)^{i}\right)\right)$ & $\frac{q^{p^{\prime}}+1}{(q+1)\left(p^{\prime}, q+1\right)}$ \\
\hline${ }^{2} A_{p^{\prime}}(q)$ & $\begin{array}{c}(q+1) \mid\left(p^{\prime}+1\right) \\
\left(p^{\prime}, q\right) \neq(3,3),(5,2)\end{array}$ & $\pi\left(q\left(q^{p^{\prime}+1}-1\right) \prod_{i=1}^{p^{\prime}-1}\left(q^{i}-(-1)^{i}\right)\right)$ & $\frac{q^{p^{\gamma}}+1}{q+1}$ \\
\hline${ }^{2} A_{3}(2)$ & & $\{2,3\}$ & 5 \\
\hline$B_{n}(q)$ & $n=2^{m} \geqslant 4, q$ нечетно & $\pi\left(q \prod_{i=1}^{n-1}\left(q^{2 i}-1\right)\right)$ & $\frac{q^{n}+1}{2}$ \\
\hline$B_{p^{\prime}}(3)$ & & $\pi\left(3\left(3^{p^{\prime}}+1\right) \prod_{i=1}^{p^{\prime}-1}\left(3^{2 i}-1\right)\right)$ & \\
\hline$C_{n}(q)$ & $n=2^{m} \geqslant 2$ & $\pi\left(q \prod_{i=1}^{n-1}\left(q^{2 i}-1\right)\right)$ & $\frac{q^{n^{-}}+1}{(2, q-1)}$ \\
\hline$C_{p^{\prime}}(q)$ & $q=2,3$ & $\pi\left(q\left(q^{p^{\prime}}+1\right) \prod_{i=1}^{p^{\prime}-1}\left(q^{2 i}-1\right)\right)$ & $\frac{q^{p^{\prime}}-1}{(2, q-1)}$ \\
\hline$D_{p^{\prime}}(q)$ & $p^{\prime} \geqslant 5, q=2,3,5$ & $\pi\left(q \prod_{i=1}^{p^{\prime}-1}\left(q^{2 i}-1\right)\right)$ & $\frac{q^{p^{\dagger}}-1}{q-1}$ \\
\hline$D_{p^{\prime}+1}(q)$ & $q=2,3$ & $\pi\left(q\left(q^{p^{\prime}}+1\right) \prod_{i=1}^{p^{\prime}-1}\left(q^{2 i}-1\right)\right)$ & $\frac{q^{p^{\prime}}-1}{(2, q-1)}$ \\
\hline${ }^{2} D_{n}(q)$ & $n=2^{m} \geqslant 4$ & $\pi\left(q \prod_{i=1}^{n-1}\left(q^{2 i}-1\right)\right)$ & $\frac{q^{n^{2}}+1}{(2, q+1)}$ \\
\hline${ }^{2} D_{n}(2)$ & $n=2^{m}+1, m \geqslant 2$ & $\pi\left(2\left(2^{n}+1\right) \prod_{i=1}^{n-2}\left(2^{2 i}-1\right)\right)$ & $2^{n-1}+1$ \\
\hline${ }^{2} D_{p^{\prime}}(3)$ & $5 \leqslant p^{\prime} \neq 2^{m}+1$ & $\pi\left(3 \prod_{i=1}^{p^{\prime}-1}\left(3^{2 i}-1\right)\right)$ & $3^{p^{\prime}}+1$ \\
\hline${ }^{2} D_{n}(3)$ & $n=2^{m}+1 \neq p^{\prime}, m \geqslant 2$ & $\pi\left(3\left(3^{n}+1\right) \prod_{i=1}^{n-2}\left(3^{2 i}-1\right)\right)$ & $\frac{n-1}{2}+1$ \\
\hline $\begin{array}{r}G_{2}(q) \\
{ }^{3} D_{4}(q)\end{array}$ & $2<q \equiv \varepsilon(\bmod 3), \varepsilon= \pm 1$ & $\begin{array}{c}\pi\left(q\left(q^{2}-1\right)\left(q^{3}-\varepsilon\right)\right) \\
\pi\left(q\left(q^{6}-1\right)\right)\end{array}$ & $\begin{array}{l}q^{2}-\varepsilon q+1 \\
q^{4}-q^{2}+1\end{array}$ \\
\hline $\begin{array}{c}F_{4}(q) \\
{ }^{2} F_{4}(2)^{\prime}\end{array}$ & $q$ нечетно & $\begin{array}{c}\pi\left(q\left(q^{6}-1\right)\left(q^{8}-1\right)\right) \\
\{2,3,5\}\end{array}$ & $\begin{array}{c}q^{4}-q^{2}+1 \\
13\end{array}$ \\
\hline$E_{6}(q)$ & & $\pi\left(q\left(q^{5}-1\right)\left(q^{8}-1\right)\left(q^{12}-1\right)\right)$ & $\frac{q^{6}+q^{3}+1}{(3, q-1)}$ \\
\hline${ }^{2} E_{6}(q)$ & $q>2$ & $\pi\left(q\left(q^{5}+1\right)\left(q^{8}-1\right)\left(q^{12}-1\right)\right)$ & $\frac{q^{6}-q^{3}+1}{(3, q+1)}$ \\
\hline$M_{12}$ & & $\{2,3,5\}$ & 11 \\
\hline$J_{2}$ & & $\{2,3,5\}$ & 7 \\
\hline$R u$ & & $\{2,3,5,7,13\}$ & 29 \\
\hline $\mathrm{He}$ & & $\{2,3,5,7\}$ & 17 \\
\hline$M c L$ & & $\{2,3,5,7\}$ & 11 \\
\hline $\mathrm{Co}_{1}$ & & $\{2,3,5,7,11,13\}$ & 23 \\
\hline $\mathrm{Co}_{3}$ & & $\{2,3,5,7,11\}$ & 23 \\
\hline$F i_{22}$ & & $\{2,3,5,7,11\}$ & 13 \\
\hline$F_{5}$ & & $\{2,3,5,7,11\}$ & 19 \\
\hline
\end{tabular}

ДокАЗАтельство. (а) Пусть $l=4 k+3$, где $k$-целое число. Тогда $2^{l}=2^{4 k+3}=$ $2^{4 k} \cdot 2^{3} \equiv 3(\bmod 5), p=2^{l}-1 \equiv 2(\bmod 5), p^{2}+1 \equiv 0(\bmod 5), r \equiv 0(\bmod 5), r=5$, $p=3$.

Пусть $l=4 k+1$, где $k$ - целое число. Тогда $p-1=2^{l}-2=2\left(2^{4 k}-1\right)=30 t_{1}$, где $t_{1}-$ целое число, поэтому $r-1=(p-1) \cdot 2^{l-1}=30 t$, где $t$ - целое число. По теореме Ферма 
$2^{l} \equiv 2(\bmod l), p=2^{l}-1 \equiv 1(\bmod l), r=\left(p^{2}+1\right) / 2 \equiv 1(\bmod l)$, т.е. $r=1+2 l s$, где $s$ - целое число. Следовательно, $30 t=2 l s, l=15 t / s-$ простое число. Ввиду простоты $l$ либо $l=3$, либо $l=5$, либо $t$ - простое число. Если $l=3$, то $p=7, r=25$ - не простое число; противоречие. Если $l=5$, то $p=31, r=481=13 \cdot 37$ - не простое число; противоречие. Значит, $t$ - простое число. Так как $2^{4 k+1}\left(2^{4 k}-1\right)=(p-1) \cdot 2^{l-1}=30 t$, то $t=2^{4 k}\left(2^{4 k}-1\right) / 15$ - не простое число; противоречие.

(б) Так как $p=2^{2^{n}}+1$, то

$$
\frac{p^{2}+1}{2}=2^{2^{n+1}-1}+2^{2^{n}}+1=2^{2^{n}-1}\left(2^{2^{n}}-1\right)+3 \cdot 2^{2^{n}-1}+1 .
$$

Если $n=0$, то $p=3, r=5$. Если $n=1$, то $p=5, r=13$. Пусть теперь $n \geqslant 2$. Тогда $2^{n}$ делится на 4 . Отсюда $2^{2^{n}}-1 \equiv 0(\bmod 5), 2^{2^{n}-1} \equiv 3(\bmod 5), 3 \cdot 2^{2^{n}-1} \equiv 4(\bmod 5)$, т.е. $\left(p^{2}+1\right) / 2 \equiv 0(\bmod 5), r \equiv 0(\bmod 5), r=5, p=3$.

3. Доказательство теоремы. Пусть $S$ - конечная простая неабелева група и $G$ конечная группа с $\omega(G)=\omega(S)$, для которой выполняется случай (а) или (б) теоремы Грюнберга-Кегеля. Ввиду лемм $1(1), 1(5)$ и 2 имеем $t(S)=2$ и, следовательно, по лемме 3 группа $S$ такова, как в таблице 1.

Лемма 9. Группа $S$ изоморфна группе лиева типа из таблицы 1.

ДокАЗАТЕЛЬСТво. Предположим противное. По лемме 4 имеем $S \cong J_{2}$. По [5] $\omega(G)=\{15,12,10,8,7\}$.

Пусть $G=F C$ - группа Фробениуса. Из леммы $1(1)$ следует, что $F-7$-групша, $C-$ группа четного порядка. По лемме $1(3)$ в $C$ есть центральный элемент порядка 2 , поэтому $2 \cdot 15=30 \in \omega(G)$; противоречие.

Пусть $G=A B C$ - двойная группа Фробениуса. Из леммы 2 следует, что $B$ - циклическая групша порядка $7,|C|$ делит 6 , поэтому $4,5 \in \omega(A)$. Так как по лемме $1(1) A$ нильпотентна, то $20 \in \omega(G)$; противоречие.

Лемма 10. Группа $S$ изоморфна $L_{3}^{\delta}(q)$ для нечетного $q$, или $S_{4}(q)$ для $(3, q)=1$, или $S_{4}(3)$. Более того, существуют числа $p_{1}, p_{2} \in \pi_{1}(S) \backslash\{p\}$ такие, что $\left\{p_{1}, p_{2}\right\} \neq$ $\{3,5\}, p_{1} p_{2} \notin \omega(G)$.

ДокАЗАТЕльство. По лемме 9 группа $S$ изоморфна группе лиева типа из таблишы. Используем классификацию и формулы для порядков максимальных торов в конечных простых группах лиева типа (см. [20, E], [21], [22, лемма 2], [23]).

1) Случай $S \cong A_{p^{\prime}-1}(q)$. Порядки максимальных торов $T$ в $S$ имеют вид

$$
|T|=\frac{1}{d} \prod_{r=1}^{m} \frac{q^{n_{r}}-1}{q-1}
$$

где $\sum_{r=1}^{m} n_{r}=p^{\prime}, d=\left(p^{\prime}, q-1\right)$.

Если $p^{\prime}=3$, то утверждение леммы следует из леммы 4 .

Пусть $p^{\prime} \geqslant 5,\left(p^{\prime}, q\right) \neq(7,2),(5,4)$. По лемме 6 числа $q^{p^{\prime}-1}-1$ и $q^{p^{\prime}-2}-1$ имеют примитивные простые делители $p_{1}$ и $p_{2}$ соответственно. Если $q=3^{f}$, то $p_{1} \neq 3, p_{2} \neq 3$. Если $q \neq 3^{f}$, то $3 \mid\left(q^{2}-1\right)$ и 3 не может быть примитивным делителем чисел $q^{p^{\prime}-1}-1$ и $q^{p^{\prime}-2}-1$ при $p^{\prime} \geqslant 5$. Поэтому $\left\{p_{1}, p_{2}\right\} \neq\{3,5\}$. Если в $S$ есть элемент порядка $p_{1} p_{2}$, то 
он по $[20, \mathrm{E}]$ содержится в максимальном торе $T$ из $S$. Так как $p_{1}, p_{2}$ не делят $\prod_{i=1}^{p^{\prime}-3}\left(q^{i}-\right.$ 1), то порядок тора $T$ должен содержать множители $\left(q^{p^{\prime}-1}-1\right) /(q-1)$ и $\left(q^{p^{\prime}-2}-1\right) /(q-$ 1). По лемме 7 имеем $\left(q^{p^{\prime}-1}-1, q^{p^{\prime}-2}-1\right)=q^{\left(p^{\prime}-1, p^{\prime}-2\right)}-1=q-1$. Отсюда

$$
\left(\frac{q^{p^{\prime}-1}-1}{q-1}, \frac{q^{p^{\prime}-2}-1}{q-1}\right)=1,
$$

поэтому порядок тора $T$ делится на

$$
\frac{\left(q^{p^{\prime}-1}-1\right)\left(q^{p^{\prime}-2}-1\right)}{(q-1)^{2} d}
$$

но $2 p^{\prime}-3>p^{\prime}$ при $p^{\prime}>3$ в противоречии с тем, что $\sum n_{r}=p^{\prime}$. Значит, в $S$ нет элемента порядка $p_{1} p_{2} ;$ противоречие с леммой $1(5)$.

Если $\left(p^{\prime}, q\right)=(7,2)$, то $\pi_{1}(S)=\{2,3,5,7,31\}$. Пусть в $S$ есть элемент порядка $7 \cdot 31$. Тогда он содержится в максимальном торе $T$,порядок которого делится на $2^{3}-1$ и $2^{5}-1$, но $3+5=8>7=p^{\prime}$; противоречие с описанием порядков максимальных торов. Значит, в $S$ нет элемента порядка $7 \cdot 31 ;$ противоречие с леммой 1 (5).

Если $\left(p^{\prime}, q\right)=(5,4)$, то $\pi_{1}(S)=\{2,3,5,7,17\}$. Пусть в $S$ есть элемент порядка $7 \cdot 17$. Тогда он содержится в максимальном торе $T$, порядок которого делится на $2^{3}-1$ и $2^{8}-1$, но $3+8=11>5=p^{\prime}$; противоречие с описанием порядков максимальных торов. Значит, в $S$ нет элемента порядка $7 \cdot 17$; противоречие с леммой $1(5)$.

2) Случай $S \cong A_{p^{\prime}}(q),(q-1) \mid\left(p^{\prime}+1\right)$. Порядки максимальных торов $T$ в $S$ имеют вид

$$
|T|=\frac{1}{d} \prod_{r=1}^{m} \frac{q^{n_{r}}-1}{q-1},
$$

где $\sum_{r=1}^{m} n_{r}=p^{\prime}+1$ и $d=\left(p^{\prime}+1, q-1\right)=q-1$.

Пусть $p^{\prime} \geqslant 5,\left(p^{\prime}, q\right) \neq(5,2),(7,2)$. По лемме 6 числа $q^{p^{\prime}+1}-1$ и $q^{p^{\prime}-1}-1$ имеют примитивные делители $p_{1}$ и $p_{2}$ соответственно. Если в $S$ есть элемент порядка $p_{1} p_{2}$, то он содержится в максимальном торе $T$ и порядок этого максимального тора делится на

$$
\frac{\left(q^{p^{\prime}+1}-1\right)\left(q^{p^{\prime}-1}-1\right)}{(q-1)^{2} d}
$$

но $\left(p^{\prime}+1\right)+\left(p^{\prime}-1\right)=2 p^{\prime}>p^{\prime}+1$; противоречие. Значит, в $S$ нет элемента порядка $p_{1} p_{2}$; противоречие с леммой $1(5)$.

При $\left(p^{\prime}, q\right)=(5,2)$ имеем $\pi_{1}(S)=\{2,3,5,7\}$. Если в $S$ есть элемент порядка $5 \cdot 7$, то он содержится в максимальном торе $T$ групшы $G$ и $|T|$ делится на $\left(2^{3}-1\right)\left(2^{4}-1\right)$, но $3+4=7>6$; противоречие. Значит, в $S$ нет элемента порядка $5 \cdot 7$; противоречие с леммой $1(5)$.

При $\left(p^{\prime}, q\right)=(7,2)$ имеем $\pi_{1}(S)=\{2,3,5,7,17,31\}$. Если в $S$ есть элемент порядка $17 \cdot 31$, то он содержится в максимальном торе $T$ грушшы $S$ и $|T|$ делится на $\left(2^{5}-1\right)\left(2^{8}-1\right)$, но $5+8=13>8$; противоречие. Значит, в $S$ нет элемента порядка $17 \cdot 31 ;$ противоречие с леммой 1 (5).

Значит, $p^{\prime}=3$ и $(q-1) \mid\left(p^{\prime}+1\right)=4$, т.е. $q=2,3,5$. По лемме 4 имеем $q \neq 3$. Если $q=2$, то $S \cong L_{4}(2)$; в частности, 2 и 5 не смежны, что противоречит лемме $1(5)$. 
Поэтому $q=5, S \cong L_{4}(5)$. По лемме $1(5) 13 \cdot 5 \in \omega(G)$, но в групше $L_{4}(5)$ нет элемента такого порядка; противоречие.

3) Случай $S \cong{ }^{2} A_{p^{\prime}-1}(q)$. Порядки максимальных торов $T$ в $S$ имеют вид

$$
|T|=\frac{1}{d} \prod_{r=1}^{m} \frac{q^{n_{r}}-(-1)^{n_{r}}}{q+1},
$$

где $\sum_{r=1}^{m} n_{r}=p^{\prime}$ и $d=\left(p^{\prime}, q+1\right)$.

Если $p^{\prime}=3$, то утверждение леммы следует из леммы 4 .

Пусть $p^{\prime} \geqslant 11$. Тогда $2\left(p^{\prime}-4\right)>p^{\prime}-1$. По лемме 6 числа $q^{2\left(p^{\prime}-2\right)}-1$ и $q^{2\left(p^{\prime}-4\right)}-1$ имеют примитивные простые делители $p_{1}$ и $p_{2}$ соответственно; в частности, $p_{1}$ делит $q^{p^{\prime}-2}+1, p_{2}$ делит $q^{p^{\prime}-4}+1$. Если в $S$ есть элемент порядка $p_{1} p_{2}$, то он содержится в максимальном торе $T$. Так как $p_{1}, p_{2}$ не делят

$$
\prod_{i=1}^{p^{\prime}-5}\left(q^{i}-(-1)^{i}\right)\left(q^{p^{\prime}-3}-1\right)\left(q^{p^{\prime}-1}-1\right)
$$

то порядок тора $T$ должен содержать множители $\left(q^{p^{\prime}-2}+1\right) /(q+1)$ и $\left(q^{p^{\prime}-4}+1\right) /(q+1)$. По лемме 7 имеем

$$
\left(q^{2\left(p^{\prime}-2\right)}-1, q^{2\left(p^{\prime}-4\right)}-1\right)=q^{2\left(p^{\prime}-2, p^{\prime}-4\right)}-1=q^{2}-1,
$$

поэтому порядок тора $T$ делится на

$$
\frac{\left(q^{p^{\prime}-2}+1\right)\left(q^{p^{\prime}-4}+1\right)}{(q+1)^{2} d}
$$

но $\left(p^{\prime}-2\right)+\left(p^{\prime}-4\right)=2 p^{\prime}-6>p^{\prime}$ при $p^{\prime} \geqslant 7$; противоречие. Значит, в $S$ нет элемента порядка $p_{1} p_{2} ;$ противоречие с леммой $1(5)$.

Пусть $p^{\prime}=7, q \neq 2$. Тогда $\pi_{1}(S)=\pi\left(q\left(q^{6}-1\right)\left(q^{5}+1\right)\left(q^{4}-1\right)\right)$. По лемме 6 числа $q^{10}-1$ и $q^{6}-1$ имеют примитивные делители $p_{1}$ и $p_{2}$ соответственно. Если $p_{2} \mid\left(q^{10}-1\right)$, то по лемме 7 получаем, что $p_{2}$ делит $\left(q^{6}-1, q^{10}-1\right)=q^{2}-1$, что противоречит определению $p_{2}$. Значит, $p_{2}$ не делит $q^{10}-1$. Пусть в $S$ есть элемент порядка $p_{1} p_{2}$. Тогда он содержится в максимальном торе $T$ группы $S$. Так как $p_{1}, p_{2}$ не делит $\left(q^{2}-1\right)\left(q^{4}-1\right)$, то порядок тора $T$ должен содержать множители $\left(q^{3}+1\right) /(q+1)$ и $\left(q^{5}+1\right) /(q+1)$. По лемме 7 имеем $\left(q^{6}-1, q^{10}-1\right)=q^{2}-1$, поэтому порядок тора $T$ делится на

$$
\frac{\left(q^{3}+1\right)\left(q^{5}+1\right)}{(q+1)^{2} d}
$$

но $3+5=8>7=p^{\prime}$; противоречие с описанием порядков максимальных торов. Значит, в $S$ нет элемента порядка $p_{1} p_{2}$; противоречие с леммой $1(5)$.

Если $\left(p^{\prime}, q\right)=(7,2)$, то $\pi_{1}(S)=\{2,3,5,7,11\}$. Предположим, что в $S$ есть элемент порядка $5 \cdot 11$. Тогда он содержится в максимальном торе $T$ и порядок $T$ делится на $\left(2^{4}-\right.$ 1) $\left(2^{5}+1\right) / 9$, но $4+5=9>7$; противоречие. Значит, в $S$ нет элемента порядка $5 \cdot 11$; противоречие с леммой $1(5)$. 
Пусть $p^{\prime}=5, q \neq 2$. Тогда $\pi_{1}(S)=\pi\left(q\left(q^{4}-1\right)\left(q^{3}+1\right)\right)$. По лемме 6 числа $q^{6}-1$ и $q^{4}-1$ имеют примитивные делители $p_{1}$ и $p_{2}$ соответственно; в частности, $p_{1}$ делит $q^{3}+1$. Если в $S$ есть элемент порядка $p_{1} p_{2}$, то он содержится в максимальном торе $T$. Порядок тора $T$ должен содержать множители $\left(q^{3}+1\right) /(q+1)$ и $\left(q^{4}-1\right) /(q+1)$. По лемме 7 имеем $\left(q^{4}-1, q^{6}-1\right)=q^{2}-1$, поэтому порядок тора $T$ делится на

$$
\frac{\left(q^{3}+1\right)\left(q^{4}-1\right)}{(q+1)^{2} d}
$$

но $3+4=7>5=p^{\prime} ;$ противоречие с описанием порядков максимальных торов. Значит, в $S$ нет элемента порядка $p_{1} p_{2}$; противоречие с леммой $1(5)$.

Если $\left(p^{\prime}, q\right)=(5,2)$, то по [5] $\mu_{1}\left(U_{5}(2)\right)=\{18,15,8\}$, поэтому $2 \cdot 5=10 \notin \omega(G)$; противоречие с леммой $1(5)$.

4) Случай $S \cong{ }^{2} A_{p^{\prime}}(q),(q+1) \mid\left(p^{\prime}+1\right),\left(p^{\prime}, q\right) \neq(3,3),(5,2)$. Порядки максимальных торов $T$ в $S$ имеют вид

$$
|T|=\frac{1}{d} \prod_{r=1}^{m} \frac{q^{n_{r}}-(-1)^{n_{r}}}{q+1},
$$

где $\sum_{r=1}^{m} n_{r}=p^{\prime}+1$ и $d=\left(p^{\prime}+1, q+1\right)=q+1$.

Пусть $p^{\prime} \geqslant 11$. Тогда $2\left(p^{\prime}-4\right)>p^{\prime}+1$. По лемме 6 числа $q^{2\left(p^{\prime}-2\right)}-1$ и $q^{2\left(p^{\prime}-4\right)}-1$ имеют примитивные простые делители $p_{1}$ и $p_{2}$ соответственно; в частности, $p_{1}$ делит $q^{p^{\prime}-2}+1, p_{2}$ делит $q^{p^{\prime}-4}+1$. Если в $S$ есть элемент порядка $p_{1} p_{2}$, то он содержится в максимальном торе $T$ из $S$. Так как $p_{1}, p_{2}$ не делят

$$
\left(q^{p^{\prime}+1}-1\right) \prod_{i=1}^{p^{\prime}-5}\left(q^{i}-(-1)^{i}\right)\left(q^{p^{\prime}-3}-1\right)\left(q^{p^{\prime}-1}-1\right),
$$

то порядок тора $T$ должен содержать множители $\left(q^{p^{\prime}-2}+1\right) /(q+1)$ и $\left(q^{p^{\prime}-4}+1\right) /(q+1)$. По лемме 7 имеем $\left(q^{2\left(p^{\prime}-2\right)}-1, q^{2\left(p^{\prime}-4\right)}-1\right)=q^{2}-1$, поэтому порядок тора $T$ делится на

$$
\frac{\left(q^{p^{\prime}-2}+1\right)\left(q^{p^{\prime}-4}+1\right)}{(q+1)^{2} d}
$$

но $\left(p^{\prime}-2\right)+\left(p^{\prime}-4\right)=2 p^{\prime}-6>p^{\prime}$ при $p^{\prime} \geqslant 7$; противоречие с описанием порядков максимальных торов. Значит, в $S$ нет элемента порядка $p_{1} p_{2} ;$ противоречие с леммой 1 (5).

Пусть $p^{\prime}=7$. Так как $(q+1) \mid\left(p^{\prime}+1\right)=8$, то $q=3,7$. Имеем

$$
\pi_{1}(S)=\pi\left(q\left(q^{8}-1\right)\left(q^{6}-1\right)\left(q^{5}+1\right)\right) .
$$

По лемме 6 числа $q^{10}-1$ и $q^{8}-1$ имеют примитивные делители $p_{1}$ и $p_{2}$ соответственно; в частности, $p_{1}$ делит $q^{5}+1$. Пусть в $S$ есть элемент порядка $p_{1} p_{2}$. Тогда он содержится в максимальном торе $T$ групш $S$. Так как $p_{1}, p_{2}$ не делят $\left(q^{6}-1\right)\left(q^{4}-1\right)$, то порядок тора $T$ должен содержать множители $\left(q^{5}+1\right) /(q+1)$ и $\left(q^{8}-1\right) /(q+1)$. По лемме 7 имеем $\left(q^{8}-1, q^{10}-1\right)=q^{2}-1$, поэтому порядок тора $T$ делится на

$$
\frac{\left(q^{5}+1\right)\left(q^{8}-1\right)}{(q+1)^{2} d}
$$


но $5+8=13>7=p^{\prime}$; противоречие с описанием порядков максимальных торов. Значит, в $S$ нет элемента порядка $p_{1} p_{2} ;$ противоречие с леммой $1(5)$.

Пусть $p^{\prime}=5$. Тогда $(q+1) \mid\left(p^{\prime}+1\right)=6$, т.е. $q=5$. Имеем $\pi_{1}(S)=\{2,3,5,7,13,31\}$. Пусть в $S$ есть элемент порядка $7 \cdot 13$. Тогда он содержится в максимальном торе $T$ из $S$. Порядок $T$ делится на $\left(5^{3}+1\right) /(5+1)=21$ и $\left(5^{4}-1\right) /(5+1)=4 \cdot 26$, поэтому $|T|$ делится на $\left(5^{3}+1\right)\left(5^{4}-1\right) / 6$, но $3+4=7>5$; противоречие. Значит, в $S$ нет элемента порядка $7 \cdot 13$; противоречие с леммой $1(5)$.

Пусть $p^{\prime}=3$ и $(q+1) \mid\left(p^{\prime}+1\right)=4$, т.е. $q=3$, что противоречит ограничению на $q$.

5) Случай $S \cong B_{n}(q), n=2^{m} \geqslant 4, q$ нечетно. Порядки максимальных торов $T$ в $S$ имеют вид

$$
|T|=\frac{1}{d} \prod_{i_{r}} \frac{q^{i_{r}}-1}{q-1} \prod_{j_{r}}\left(q^{j_{r}}+1\right),
$$

где $\sum i_{r}+\sum j_{r}=n$ и $d=(2, q-1)$.

По лемме 6 числа $q^{2(n-1)}-1$ и $q^{2(n-2)}-1$ имеют примитивные простые делители $p_{1}$ и $p_{2}$ соответственно; в частности, $p_{1}$ делит $q^{n-1}+1, p_{2}$ делит $q^{n-2}+1$. Так как $(n-1)+(n-2)=2 n-3>n$ при $n>3$, то $p_{1} p_{2}$ не может быть порядком полупростого элемента в $S$. Значит, в $S$ нет элемента порядка $p_{1} p_{2} ;$ противоречие с леммой $1(5)$.

6) Случай $S \cong B_{p^{\prime}}(3)$. Порядки максимальных торов $T$ в $S$ имеют вид

$$
|T|=\frac{1}{2} \prod_{i_{r}} \frac{3^{i_{r}}-1}{2} \prod_{j_{r}}\left(3^{j_{r}}+1\right),
$$

где $\sum i_{r}+\sum j_{r}=p^{\prime}$.

По лемме 6 числа $3^{2 p^{\prime}}-1$ и $3^{2\left(p^{\prime}-1\right)}-1$ имеют примитивные простые делители $p_{1}$ и $p_{2}$ соответственно; в частности, $p_{1}$ делит $3^{p^{\prime}}+1, p_{2}$ делит $3^{p^{\prime}-1}+1$. Так как $p^{\prime}+\left(p^{\prime}-1\right)=$ $2 p^{\prime}-1>p^{\prime}$, то $p_{1} p_{2}$ не может быть порядком полупростого элемента в $S$. Значит, в $S$ нет элемента порядка $p_{1} p_{2} ;$ противоречие с леммой $1(5)$.

7) Случай $S \cong C_{n}(q), n=2^{m} \geqslant 2$. Если $n=2, q=3^{f}, f>1$, то утверждение леммы следует из леммы 4 .

Пусть теперь $n \geqslant 4$.

Порядки максимальных торов $T$ в $S$ имеют вид

$$
|T|=\frac{1}{d} \prod_{i_{r}} \frac{q^{i_{r}}-1}{q-1} \prod_{j_{r}}\left(q^{j_{r}}+1\right),
$$

где $\sum i_{r}+\sum j_{r}=n$ и $d=(2, q-1)$.

Пусть $(n, q) \neq(4,2)$. По лемме 6 числа $q^{2(n-1)}-1$ и $q^{2(n-2)}-1$ имеют примитивные простые делители $p_{1}$ и $p_{2}$ соответственно; в частности, $p_{1}$ делит $q^{n-1}+1, p_{2}$ делит $q^{n-2}+1$. Так как $(n-1)+(n-2)=2 n-3>n$ при $n>3$, то $p_{1} p_{2}$ не может быть порядком полупростого элемента в $S$; противоречие с леммой $1(5)$.

Если $(n, q)=(4,2)$, то $\pi_{1}(S)=\{2,3,5,7\}$. Если есть элемент порядка $5 \cdot 7$, то он содержится в максимальном торе $T$ из $S$ и порядок этого максимального тора делится на $2^{3}-1$ и $2^{2}+1$. Но $3+2=5>4=n$; противоречие. Значит, в $S$ нет элемента порядка $5 \cdot 7$; противоречие с леммой $1(5)$. 
8) Случай $S \cong C_{p^{\prime}}(q), q=2,3$. Порядки максимальных торов $T$ в $S$ имеют вид

$$
|T|=\frac{1}{d} \prod_{i_{r}} \frac{q^{i_{r}}-1}{q-1} \prod_{j_{r}}\left(q^{j_{r}}+1\right),
$$

где $\sum i_{r}+\sum j_{r}=p^{\prime}$ и $d=(2, q-1)$.

По лемме 4 получаем $\left(p^{\prime}, q\right) \neq(3,2)$. По лемме 6 числа $q^{2 p^{\prime}}-1$ и $q^{2\left(p^{\prime}-1\right)}-1$ имеют примитивные простые делители $p_{1}$ и $p_{2}$ соответственно; в частности, $p_{1}$ делит $q^{p^{\prime}}+1$, $p_{2}$ делит $q^{p^{\prime}-1}+1$. Так как $p^{\prime}+\left(p^{\prime}-1\right)=2 p^{\prime}-1>p^{\prime}$ при $n>3$, то $p_{1} p_{2}$ не может быть порядком полупростого элемента в $S$; противоречие с леммой $1(5)$.

9) Случай $S \cong D_{p^{\prime}}(q), p^{\prime} \geqslant 5, q=2,3,5$. Порядки максимальных торов $T$ в $S$ имеют вид

$$
|T|=\frac{1}{d} \prod_{i_{r}} \frac{q^{i_{r}}-1}{q-1} \prod_{j_{r}, k_{r}}\left(q^{j_{r}}+1\right)\left(q^{k_{r}}+1\right),
$$

где $\sum i_{r}+\sum\left(j_{r}+k_{r}\right)=p^{\prime}$ и $d=\left(4, q^{p^{\prime}}-1\right)$.

Пусть $\left(p^{\prime}, q\right) \neq(5,2)$. По лемме 6 числа $q^{2\left(p^{\prime}-1\right)}-1$ и $q^{2\left(p^{\prime}-2\right)}-1$ имеют примитивные простые делители $p_{1}$ и $p_{2}$ соответственно; в частности, $p_{1}$ делит $q^{p^{\prime}-1}+1, p_{2}$ делит $q^{p^{\prime}-2}+1$. Так как $\left(p^{\prime}-1\right)+\left(p^{\prime}-2\right)=2 p^{\prime}-3>p^{\prime}$ при $p^{\prime}>3$, то $p_{1} p_{2}$ не может быть порядком полупростого элемента в $S$; противоречие с леммой $1(5)$.

Если $\left(p^{\prime}, q\right)=(5,2)$, то $\pi_{1}(S)=\{2,3,5,7,17\}$. Если есть элемент порядка $7 \cdot 17$, то он содержится в максимальном торе $T$ из $S$ и порядок этого максимального тора делится на $2^{3}-1$ и $2^{4}+1$. Но $3+4=7>5=p^{\prime}$; противоречие. Значит, в $S$ нет элемента порядка $7 \cdot 17$; противоречие с леммой $1(5)$.

10) Случай $S \cong D_{p^{\prime}+1}(q), q=2,3$. Порядки максимальных торов $T$ в $S$ имеют вид

$$
|T|=\frac{1}{d} \prod_{i_{r}} \frac{q^{i_{r}}-1}{q-1} \prod_{j_{r}, k_{r}}\left(q^{j_{r}}+1\right)\left(q^{k_{r}}+1\right),
$$

где $\sum i_{r}+\sum\left(j_{r}+k_{r}\right)=p^{\prime}+1$ и $d=\left(4, q^{p^{\prime}+1}-1\right)$.

По лемме $4\left(p^{\prime}, q\right) \neq(3,2)$. По лемме 6 числа $q^{2 p^{\prime}}-1$ и $q^{2\left(p^{\prime}-1\right)}-1$ имеют примитивные простые делители $p_{1}$ и $p_{2}$ соответственно; в частности, $p_{1}$ делит $q^{p^{\prime}}+1, p_{2}$ делит $q^{p^{\prime}-1}+$ 1. Так как $p^{\prime}+\left(p^{\prime}-1\right)=2 p^{\prime}-1>p^{\prime}+1$, то $p_{1} p_{2}$ не может быть порядком полупростого элемента в $S$. Значит, в $S$ нет элемента порядка $p_{1} p_{2} ;$ противоречие с леммой $1(5)$.

11) Случай $S \cong{ }^{2} D_{n}(q), n=2^{m} \geqslant 4$. Порядки максимальных торов $T$ в $S$ имеют вид

$$
|T|=\frac{1}{d} \prod_{i_{r}} \frac{q^{i_{r}}-(-1)^{i_{r}}}{q+1} \prod_{j_{r}, k_{r}}\left(q^{j_{r}}+(-1)^{j_{r}}\right)\left(q^{k_{r}}+(-1)^{k_{r}}\right),
$$

где $\sum i_{r}+\sum\left(j_{r}+k_{r}\right)=n$ и $d=\left(4, q^{n}+1\right)$.

Пусть $(n, q) \neq(4,2)$. По лемме 6 числа $q^{2(n-1)}-1$ и $q^{2(n-2)}-1$ имеют примитивные простые делители $p_{1}$ и $p_{2}$ соответственно; в частности, $p_{1}$ делит $q^{n-1}+1, p_{2}$ делит $q^{n-2}+1$. Так как $(n-1)+(n-2)=2 n-3>n$ при $n>3$, то $p_{1} p_{2}$ не может быть порядком полупростого элемента в $S$; противоречие с леммой $1(5)$.

Если $(n, q)=(4,2)$, то по [5] $\mu_{1}(S)=\{30,21,12,9,8\}$, поэтому в $S$ нет элемента порядка $7 \cdot 5$; противоречие с леммой 1 (5). 
12) Случай $S \cong{ }^{2} D_{n}(2), n=2^{m}+1, m \geqslant 2$. Порядки максимальных торов $T$ в $S$ имеют вид

$$
|T|=\prod_{i_{r}} \frac{2^{i_{r}}-(-1)^{i_{r}}}{3} \prod_{j_{r}, k_{r}}\left(2^{j_{r}}+(-1)^{j_{r}}\right)\left(2^{k_{r}}+(-1)^{k_{r}}\right),
$$

где $\sum i_{r}+\sum\left(j_{r}+k_{r}\right)=n$.

Пусть $(n, q) \neq(5,2)$. По лемме 6 числа $2^{2 n}-1$ и $2^{2(n-2)}-1$ имеют примитивные простые делители $p_{1}$ и $p_{2}$ соответственно; в частности, $p_{1}$ делит $2^{n}+1, p_{2}$ делит $2^{n-2}+1$. Так как $n+(n-2)=2 n-2>n$ при $n>2$, то $p_{1} p_{2}$ не может быть порядком полупростого элемента в $S$; противоречие с леммой $1(5)$.

Если $(n, q)=(5,2)$, то по [5] $\mu_{1}(S)=\{35,33,30,24,21,20,18,14,8\}$, поэтому в $S$ нет элемента порядка $7 \cdot 11$; противоречие с леммой $1(5)$.

13) Случай $S \cong{ }^{2} D_{p^{\prime}}(3), 5 \leqslant p^{\prime} \neq 2^{m}+1$. Порядки максимальных торов $T$ в $S$ имеют вид

$$
|T|=\frac{1}{d} \prod_{i_{r}} \frac{3^{i_{r}}-(-1)^{i_{r}}}{4} \prod_{j_{r}, k_{r}}\left(3^{j_{r}}+(-1)^{j_{r}}\right)\left(3^{k_{r}}+(-1)^{k_{r}}\right),
$$

где $\sum i_{r}+\sum\left(j_{r}+k_{r}\right)=p^{\prime}$ и $d=\left(4,3^{p^{\prime}}+1\right)$.

По лемме 6 числа $3^{2\left(p^{\prime}-1\right)}-1$ и $3^{2\left(p^{\prime}-2\right)}-1$ имеют примитивные простые делители $p_{1}$ и $p_{2}$ соответственно; в частности, $p_{1}$ делит $3^{p^{\prime}-1}+1, p_{2}$ делит $3^{p^{\prime}-2}+1$. Так как $\left(p^{\prime}-1\right)+\left(p^{\prime}-2\right)=2 p^{\prime}-3>p^{\prime}$ при $p^{\prime}>3$, то $p_{1} p_{2}$ не может быть порядком полупростого элемента в $S$; противоречие с леммой $1(5)$.

14) Случай $S \cong{ }^{2} D_{n}(3), n=2^{m}+1 \neq p^{\prime}, m \geqslant 2$. Порядки максимальных торов $T$ в $S$ имеют вид

$$
|T|=\frac{1}{4} \prod_{i_{r}} \frac{3^{i_{r}}-(-1)^{i_{r}}}{4} \prod_{j_{r}, k_{r}}\left(3^{j_{r}}+(-1)^{j_{r}}\right)\left(3^{k_{r}}+(-1)^{k_{r}}\right)
$$

где $\sum i_{r}+\sum\left(j_{r}+k_{r}\right)=n$.

По лемме 6 числа $3^{2 n}-1$ и $3^{2(n-2)}-1$ имеют примитивные простые делители $p_{1}$ и $p_{2}$ соответственно; в частности, $p_{1}$ делит $3^{n}+1, p_{2}$ делит $3^{n-2}+1$. Так как $n+(n-2)=$ $2 n-2>n$ при $n>2$, то $p_{1} p_{2}$ не может быть порядком полупростого элемента в $S$; противоречие с леммой $1(5)$.

15) Случай $S \cong G_{2}(q), 2<q \equiv \varepsilon(\bmod 3), \varepsilon= \pm 1$. Максимальные торы $T$ в $S$ имеют следующие порядки:

$$
(q-1)^{2}, \quad q^{2}-1, \quad q^{2}-q+1, q^{2}+q+1, \quad(q+1)^{2}
$$

Пусть $q \neq 4, \varepsilon=1$. По лемме 6 числа $q^{2}-1$ и $q^{3}-1$ имеют примитивные простые делители $p_{1}$ и $p_{2}$ соответственно. Так как $p_{1} p_{2}$ не делит порядок максимального тора $T$, то в $S$ нет элемента порядка $p_{1} p_{2} ;$ противоречие с леммой 1 (5).

Если $q=4$, то по [5] $\mu_{1}(S)=\{7 \cdot 3,5 \cdot 3,5 \cdot 2,3 \cdot 4,8\}$, поэтому в $S$ нет элемента порядка $5 \cdot 7$; противоречие с леммой $1(5)$.

Пусть $\varepsilon=-1, p_{1} \in \pi(q-1)$. По лемме 6 число $q^{6}-1$ имеет примитивньй простой делитель $p_{2}$. Так как $p_{1} p_{2}$ не делит порядок максимального тора $T$, то в $S$ нет элемента порядка $p_{1} p_{2} ;$ противоречие с леммой 1 (5). 
16) Случай $S \cong{ }^{3} D_{4}(q)$. Максимальные торы $T$ в $S$ имеют следующие порядки:

$$
\begin{gathered}
\left(q^{3}+1\right)(q+1), \quad\left(q^{3}-1\right)(q+1), \quad\left(q^{3}-1\right)(q-1), \quad\left(q^{3}+1\right)(q-1), \\
\left(q^{2}+q+1\right)^{2}, \quad\left(q^{2}-q+1\right)^{2}, q^{4}-q^{2}+1 .
\end{gathered}
$$

По лемме 4 имеем $q \neq 2$. Пусть $q \neq 4$. По лемме 6 числа $q^{3}-1$ и $q^{6}-1$ имеют примитивные простые делители $p_{1}$ и $p_{2}$ соответственно; в частности, $p_{1}$ не делит $q^{2}-1$, $p_{2}$ делит $q^{3}+1$, поэтому в $S$ нет элемента порядка $p_{1} p_{2}$; противоречие с леммой 1 (5).

Если $q=4$, то $q^{3}+1=65, q^{3}-1=63$, поэтому в $S$ нет элемента порядка $7 \cdot 13$; противоречие с леммой $1(5)$.

17) Случай $S \cong F_{4}(q), q$ нечетно. Максимальные торы $T$ в $S$ имеют следующие порядки:

$(q-1)^{4}, \quad\left(q^{2} \pm 1\right)(q \pm 1)^{2}, \quad\left(q^{2}-1\right)^{2}, \quad\left(q^{3} \pm 1\right)(q \pm 1), \quad(q+1)^{4}, \quad q^{4}-1, \quad q^{4}+1, \quad\left(q^{2}+1\right)^{2}$.

По лемме 6 числа $q^{6}-1$ и $q^{8}-1$ имеют примитивные простые делители $p_{1}$ и $p_{2}$ соответственно, поэтому $p_{1}$ делит $q^{3}+1, p_{2}$ делит $q^{4}+1$. Таким образом, в $S$ нет элемента порядка $p_{1} p_{2} ;$ противоречие с леммой 1 (5).

18) Случай $S \cong{ }^{2} F_{4}(2)^{\prime}$. По лемме 4 этот случай невозможен.

19) Случай $S \cong E_{6}(q)$. Пусть $d=(3, q-1)$. Тогда максимальные торы $T$ в $S$ имеют следуюшие порядки:

$$
\begin{gathered}
\frac{(q-1)^{6}}{d}, \frac{(q+1)(q-1)^{5}}{d}, \frac{(q+1)^{2}(q-1)^{4}}{d}, \frac{(q-1)^{3}\left(q^{3}-1\right)}{d}, \frac{\left(q^{2}-1\right)^{3}}{d} \\
\frac{\left(q^{3}-1\right)\left(q^{2}-1\right)(q \pm 1)}{d}, \frac{\left(q^{4}-1\right)(q \pm 1)^{2}}{d}, \frac{\left(q^{2}-1\right)^{2}(q+1)^{2}}{d}, \frac{\left(q^{3}-1\right)^{2}}{d} \\
\frac{\left(q^{4} \pm 1\right)\left(q^{2}-1\right)}{d}, \frac{\left(q^{5}-1\right)(q \pm 1)}{d}, \frac{\left(q^{3}+1\right)\left(q^{2} \pm 1\right)(q-1)}{d}, \frac{\left(q^{2}+1\right)^{2}(q-1)^{2}}{d} \\
\frac{\left(q^{2}+q+1\right)^{2}\left(q^{2}-1\right)}{d}, \frac{\left(q^{6}-1\right)}{d}, \frac{\left(q^{2}+q+1\right)^{3}}{d}, \frac{\left(q^{3}+1\right)\left(q^{2}+q+1\right)(q+1)}{d} \\
\frac{\left(q^{4}-q^{2}+1\right)\left(q^{2}+q+1\right)}{d}, \frac{\left(q^{6}+q^{3}+1\right)}{d}, \frac{\left(q^{2}+q+1\right)\left(q^{2}-q+1\right)^{2}}{d}
\end{gathered}
$$

По лемме 6 числа $q^{12}-1$ и $q^{8}-1$ имеют примитивные простые делители $p_{1}$ и $p_{2}$ соответственно. Таким образом, в $S$ нет элемента порядка $p_{1} p_{2} ;$ противоречие с леммой $1(5)$.

20) Случай $S \cong{ }^{2} E_{6}(q), q>2$. Пусть $d=(3, q+1)$. Тогда максимальные торы $T$ в $S$ имеют следуюшие порядки:

$$
\begin{aligned}
& \frac{(q+1)^{6}}{d}, \quad \frac{\left(q^{2}-1\right)(q+1)^{4}}{d}, \frac{\left(q^{2} \pm 1\right)^{2}(q+1)^{2}}{d}, \frac{\left(q^{3}+1\right)(q+1)^{3}}{d}, \\
& \frac{\left(q^{2}-1\right)^{3}}{d}, \frac{\left(q^{3}+1\right)\left(q^{2}-1\right)(q \pm 1)}{d}, \frac{\left(q^{4}-1\right)(q \pm 1)^{2}}{d}, \frac{\left(q^{2}-1\right)^{2}(q-1)^{2}}{d}, \\
& \frac{\left(q^{3}+1\right)^{2}}{d}, \frac{\left(q^{4} \pm 1\right)\left(q^{2}-1\right)}{d}, \frac{\left(q^{5}+1\right)(q \pm 1)}{d}, \frac{\left(q^{3}-1\right)\left(q^{2} \pm 1\right)(q+1)}{d},
\end{aligned}
$$




$$
\begin{gathered}
\frac{\left(q^{2}-q+1\right)^{2}\left(q^{2}-1\right)}{d}, \frac{\left(q^{6}-1\right)}{d}, \frac{\left(q^{2}-q+1\right)^{3}}{d}, \frac{\left(q^{3}-1\right)\left(q^{2}-q+1\right)(q-1)}{d} \\
\frac{\left(q^{4}-q^{2}+1\right)\left(q^{2}-q+1\right)}{d}, \frac{\left(q^{6}-q^{3}+1\right)}{d}, \frac{\left(q^{2}+q+1\right)^{2}\left(q^{2}-q+1\right)}{d} .
\end{gathered}
$$

По лемме 6 числа $q^{12}-1$ и $q^{8}-1$ имеют примитивные простые делители $p_{1}$ и $p_{2}$ соответственно. Таким образом, в $S$ нет элемента порядка $p_{1} p_{2}$; противоречие с леммой $1(5)$.

Лемма 11. Если $S \cong L_{3}(q)$ для нечетного $q$, mо $q=3$ и $G$ - группа Фробениуса.

ДокАЗАтЕльство. Пусть $G=F C$ - группа Фробениуса с ядром $F$ и дополнением $C$. Предположим, что $F-\pi_{1}$-группа. По лемме $1(1)$ имеем $|\mu(F)|=1$, что противоречит лемме 5 . Значит, $F-\pi_{2}$-групша, $C-\pi_{1}$-группа.

Предположим сначала, что $C$ разрешима. Тогда по лемме $1(5)$ граф $G K(C)$ полньй. Если $t \in \pi(q+1) \backslash\{2\}$, то по лемме 5 вершины $p$ и $t$ не смежны в $\pi_{1}(C)$, что противоречит лемме $1(5)$. Значит, $\pi(q+1)=\{2\}$, откуда $q=2^{n}-1=p, n$ - простое число. При $n=2$ имеем $q=3$. Пусть $n \geqslant 3$. Тогда $(3, p-1)=3$. При $n=3$ имеем $q=7$, что противоречит [12]. Поэтому можно считать, что $n \geqslant 5$. Тогда $p+1$ делится на 32 и по лемме 1 (4) $C$ имеет нормальное 2-дополнение $O(C)$. Пусть $P \in \operatorname{Syl}_{3}(O(C)),|P|=3^{m}$. По лемме $1(2) \quad P$ циклическая. По аргументу Фраттини $C=O(C) N_{C}(P)$, отсюда по лемме 5 порядок $\left|N_{C}(P)\right|$ делится на $2^{n+1}$. Так как $N_{C}(P) / C_{C}(P)$ изоморфна подгруппе из $\operatorname{Aut}(P)$ и по теореме (23.3) из [4] $\mid$ Aut $P \mid=2 \cdot 3^{m-1}$, то $\left|C_{C}(P)\right|$ делится на $2^{n}$. По лемме 1 (3) силовская 2-подгруппа в $C$ циклическая или (обобщенная) група кватернионов. Если силовская 2-подгруппа в $C$ циклическая, то в $C$ есть элемент порядка $2^{n} \cdot 3^{m}$, что противоречит лемме 5. Если силовская 2-подгруппа (обобщенная) кватернионная, то в $C$ есть элемент порядка $2^{n-1} \cdot 3^{m}$, откуда $n=2$; противоречие.

Предположим теперь, что $C$ неразрешима. Из леммы $1(5)$ следует, что 3 и 5 - единственная пара несмежных вершин в графе $G K(C)$. По лемме 5 либо $p=3$ и $5 \mid(q+1)$, либо $p=5$ и $3 \mid(q+1)$. Если $p=3$ и $5 \mid(q+1)$, то $\pi(q+1)=\{2,5\}$. По лемме $1(5)$ либо $q+1=5 \cdot 4, q=19$, либо $q+1=5 \cdot 8, q=39$. Противоречие с тем, что $q-$ степень 3 . Если $p=5$ и $3 \mid(q+1)$, то $\pi(q+1)=\{2,3\}$. По лемме $1(5)$ либо $q=3 \cdot 4, q=11$, либо $q+1=3 \cdot 8, q=23$. Противоречие с тем, что $q-$ степень 5 .

Пусть $G=A B C$, где $A, A B$ - нормальные подгрупшы группы $G, A B, B C$ - группы $\Phi$ робениуса с ядрами $A, B$ и дополнениями $B, C$ соответственно. Из лемм 2,3 следует, что $B$ - циклическая группа порядка $\left(q^{2}+q+1\right) /(3, q-1), G K(A C)$ - полньй граф. По лемме 5 получаем, что $q=2^{n}-1=p$, где $n$ - простое число. Если $n=2$, то $q=3$. Тогда $\mu(G)=\{13,3 \cdot 2,8\}$, поэтому $\operatorname{Exp} A=8, B$ - циклическая группа порядка $13, C-$ циклическая группа порядка $3, C_{A}(C)$ элементарная абелева. По лемме 7.3 из [9] класс нильпотентности $A$ не больше 2. Отсюда по лемме 2 экспонента $A$ не превосходит 4; противоречие. Значит, $n \geqslant 3$. Пусть $p \in \pi(C)$. Поскольку подгруппа $C$ действует полурегулярно на $B \backslash\{1\}$, то $p^{2}+p+1 \equiv 3(\bmod p), p=2$, что противоречит нечетности $p$. Поэтому $p \in \pi(A)$. Тогда по лемме 5 получаем, что $p+1=2^{n} \in \omega(C)$. По лемме 2 имеем $p(p+1) \in \omega(G)$; противоречие с леммой 5 .

ПримеР 1 (см. [9, предложение 3]). Существует конечная группа Фробениуса $G$ с $\omega(G)=\omega\left(L_{3}(3)\right)$. Действительно, пусть $H$ - расширение группы порядка 2 при помощи $S_{4}$ такое, что силовская 2 -подгруппа в $H$ является обобщенной группй кватерни- 
онов. Тогда $\mu(H)=\{6,8\}$. По лемме 8 из [9] существует расширение $G$ элементар-

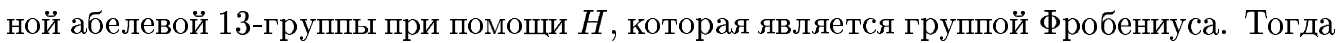
$\mu(G)=\{6,8,13\}=\mu\left(L_{3}(3)\right)$.

Лемма 12. Если $S \cong U_{3}(q)$ для нечетного $q$, то $q=3$.

ДокАЗАтЕльство. Пусть $G=F C$ - группа Фробениуса с ядром $F$ и дополнением $C$. Предположим, что $F-\pi_{1}$-группа. По лемме $1(1)$ имеем $|\mu(F)|=1$, что противоречит лемме 5 . Значит, $F-\pi_{2}$-групша, $C-\pi_{1}$-группа.

Предположим сначала, что $C$ разрешима. Тогда по лемме $1(5)$ граф $G K(C)$ полньй. Если $t \in \pi(q-1) \backslash\{2\}$, то по лемме 5 вершины $p$ и $t$ не смежны в $G K(C)$; противоречие. Значит, $\pi(q-1)=\{2\}$, откуда $q=9$ или $q=2^{2^{n}}+1=p,(3, p+1)=3$. По предложению 4 из [9] $q \neq 9$. Если $q=5$, то $\mu_{1}(G)=\{10,8,6\}$, откуда $15 \notin \omega(C)$, что противоречит полноте графа $G K(C)$. Итак, $q=2^{2^{n}}+1$, где $n \geqslant 2$. Тогда $q-1=2^{2^{n}}$ делится на 16 и лемма $1(4)$ влечет, что $C$ имеет нормальное 2 -дополнение $O(C)$. Пусть $P \in \mathrm{Syl}_{3}(O(C)),|P|=3^{m}$. Из леммы $1(2)$ следует, что $P$ циклическая. По аргументу $\Phi$ раттини $C=O(C) N_{C}(P)$, поэтому $\left|N_{C}(P)\right|$ делится на $2^{2^{n}+1}$. Так как $N_{C}(P) / C_{C}(P)$ изоморфна подгрупе из Aut $P$ и по теореме (23.3) из [4] $\mid$ Aut $P \mid=2 \cdot 3^{m-1}$, то $\left|C_{C}(P)\right|$ делится на $2^{2^{n}}$. По лемме $1(3)$ силовская 2 -подгруппа из $C$ циклическая или (обобщенная) группа кватернионов. Если силовская 2-подгруппа из $C$ циклическая, то в $C$ есть элемент порядка $2^{2^{n}} \cdot 3^{m}$, что противоречит лемме 5. Если силовская 2-подгруппа из $C$ (обобщенная) кватернионная, то в $C$ есть элемент порядка $2^{2^{n}-1} \cdot 3^{m}$, откуда $2^{n}=2$, $n=1$, но $n \geqslant 2$; противоречие.

Предположим теперь, что $C$ неразрешима. Из леммы $1(5)$ следует, что 3 и 5 - единственная пара несмежных вершин в графе $G K(C)$. По лемме 5 либо $p=3$ и $5 \mid(q-1)$, либо $p=5$ и $3 \mid(q-1)$. Если $p=3$ и $5 \mid(q-1)$, то $\pi(q-1)=\{2,5\}$. По лемме $1(5)$ либо $q-1=5 \cdot 4, q=21$, либо $q-1=5 \cdot 8, q=41$. Противоречие с тем, что $q-$ степень 3 . Если $p=5$ и $3 \mid(q-1)$, то $\pi(q-1)=\{2,3\}$. По лемме $1(5)$ либо $q-1=3 \cdot 4, q=13$, либо $q-1=3 \cdot 8, q=25$. Таким образом, $q=25$. По лемме 5 имеем $\mu(C)=\{2 \cdot 5 \cdot 13,16 \cdot 3 \cdot 13\}$, поэтому $16 \in \omega(C)$, что противоречит лемме $1(5)$.

Пусть $G=A B C$, где $A, A B$ - нормальные подгрупшы группы $G, A B, B C$ - группы $\Phi$ робениуса с ядрами $A, B$ и дополнениями $B, C$ соответственно. Из лемм 2,3 следует, что $B$ - циклическая группа порядка $\left(q^{2}-q+1\right) /(3, q+1), G K(A C)$ - полньй граф. По лемме 5 либо $q=9$, либо $q=2^{2^{n}}+1=p$. По предложению 4 из [9] $q \neq 9$. Пусть $p \in \pi(C)$. Поскольку подгруппа $C$ действует полурегулярно на $B \backslash\{1\}$, имеем $p^{2}-$ $p+1 \equiv 3(\bmod p), p=2$, что противоречит нечетности $p$. Поэтому $p \in \pi(A)$. Тогда $p-1=2^{2^{n}} \in \omega(C)$. По лемме 2 имеем $p(p-1) \in \omega(G)$; противоречие с леммой 5 .

ПримеР 2 (В.Д. Мазуров). Существует конечная группа $\omega\left(U_{3}(3)\right)$. Действительно, пусть $W$ - векторное пространство размерности 2 над полем $F$ из 49 элементов, $H$ - группа, порожденная матрищами

$$
a=\left(\begin{array}{cc}
\alpha & 0 \\
0 & 1 / \alpha
\end{array}\right), \quad b=\left(\begin{array}{cc}
0 & \beta \\
-\beta & 0
\end{array}\right),
$$

где $\alpha, \beta$ - элементы из $F^{*}$ порядка 3 и 8 соответственно. Тогда $H$ - неабелево полупрямое произведение $\langle a\rangle$ на $\langle b\rangle$. Группа $G=W H$ - группа Фробениуса с ядром $W$ и дополнением $H$, причем $\omega(G)=\omega\left(U_{3}(3)\right)$. 
Лемма 13. Группа $S$ не изоморфна $S_{4}\left(2^{f}\right)$ nри $f>1$.

ДокАЗАТЕльСтво. Предположим, что $S \cong S_{4}(q)$, где $q$ четно, $q>2$. Пусть $G=F C$ - группа Фробениуса. Из леммы $1(1)$ следует, что $F$ нильпотентна, поэтому по лемме 7 из [10] $\mu(F)=\left\{q^{2}+1\right\}, \mu(C)=\left\{4,2(q-1), 2(q+1), q^{2}-1\right\}$. По лемме $1(3)$ имеем $2\left(q^{2}-1\right) \in \omega(C) ;$ противоречие со строением $\mu(C)$.

Пусть $G=A B C$ - двойная группа $\Phi$ робениуса. Тогда леммы 2,3 влекут, что $B-$ циклическая группа порядка $q^{2}+1, C$ - циклическая группа порядка, делящего $|B|-$ $1=q^{2}$. Отсюда по лемме 7 из [10] $|C|$ делит 4 и $\mu(A)=\left\{q^{2}-1\right\}$. Если $|C|=4$ и $t \in \pi(q-1)$, то по лемме 2 имеем $4 t \in \omega(G)$; противоречие со строением $\mu(G)$. Пусть $|C|=2, t_{1} \in \pi(q-1), t_{2} \in \pi(q+1)$. Тогда по лемме 2 имеем $2 t_{1} t_{2} \in \omega(G) ;$ противоречие со строением $\mu(G)$.

Лемма 14. Группа $S$ не изоморфна $S_{4}(q)$ при $(6, q)=1$.

ДокаЗАТЕЛЬСТво. Предположим противное. Пусть $G=F C$ - група $\Phi$ робениуса. Из леммы $1(1)$ следует, что $F$ нильпотентна, поэтому по лемме 3 из $[24] \mu(F)=\left\{\left(q^{2}+\right.\right.$ $1) / 2\}, \mu(C)=\left\{\left(q^{2}-1\right) / 2, p(q+1), p(q-1)\right\}$. Групша $C$ действует полурегулярно на $F \backslash\{1\}$. Поэтому $|C|$ делит $|F \backslash\{1\}|=\left(q^{2}-1\right) / 2$; противоречие со строением $\mu(C)$.

Пусть $G=A B C$ - двойная группа Фробениуса. По лемме 3 из [24] и леммам 2,3 имеем $\mu(B)=\left\{\left(q^{2}+1\right) / 2\right\}, \mu(A C)=\left\{\left(q^{2}-1\right) / 2, p(q+1), p(q-1)\right\}$. Так как $|C|$ делит $|B|-1$, то $|C|$ делит $\left(q^{2}-1\right) / 2$, следовательно, $p \in \pi(A)$.

Пусть $p(q+1) / t \in \mu(A)$, где $t$ - целое число. По описанию $\mu(C)$ и лемме 2 имеем $t(q-1) / 2 \in \omega(C)$. По лемме 2 имеем

$$
p \cdot \frac{q-1}{2} t \cdot \prod_{s \in \pi(A) \backslash\{p\}} s \in \omega(G),
$$

откуда $t=1,2$. Если $t=2$, то $\pi(A)=\{p\}, \mu(A)=\{p\}$, но $p(q+1) / 2 \in \omega(A)$; противоречие. Итак, $t=1$. Тогда $\pi(A)=\{2, p\}, q+1$ является степенью 2 , поэтому $q=2^{n}-1=p$, где $n$-натуральное число. Отсюда $\mu(A)=\{p(p+1)\}, B$ - циклическая группа порядка $\left(p^{2}+1\right) / 2, C$ - циклическая группа порядка $(p-1) / 2$. Докажем, что группа $B$ простого порядка. Пусть это не так. Тогда $p_{1} p_{2}$ делит $|B|$, где $p_{1}, p_{2}-$ простые числа (возможно, одинаковые). Отсюда $|C|$ делит $p_{1}-1,|C|$ делит $p_{2}-1$, поэтому $p_{1}-1=|C| k_{1}=k_{1}(p-1) / 2, p_{2}-1=|C| k_{2}=k_{2}(p-1) / 2$, где $k_{1}, k_{2}$ - натуральные числа. Пусть $k_{i}=1$ для некоторого $i \in\{1,2\}$. Тогда $p_{i}=(p+1) / 2=2^{n-1}$, поэтому $|B|$ четен; противоречие. Итак, $k_{1}, k_{2} \geqslant 2$. Тогда

$$
\frac{p^{2}+1}{2}=|B| \geqslant p_{1} p_{2}=\left(\frac{p-1}{2} k_{1}+1\right)\left(\frac{p-1}{2} k_{2}+1\right) \geqslant p^{2} ;
$$

противоречие. Таким образом, $B$ простого порядка, т.е. $\left(p^{2}+1\right) / 2-$ простое число. По лемме 8 (а) имеем $p=3$; противоречие с условием.

Пусть $p(q-1) / t \in \mu(A)$, где $t$ - целое число. По описанию $\mu(C)$ и лемме 2 имеем $t(q+1) / 2 \in \omega(C)$. По лемме 2 имеем

$$
p \cdot \frac{q+1}{2} t \cdot \prod_{s \in \pi(A) \backslash\{p\}} s \in \omega(G),
$$


откуда $t=1,2$. Если $t=2$, то $\pi(A)=\{p\}, \mu(A)=\{p\}$, но $p(q-1) / 2 \in \omega(A)$; противоречие. Итак, $t=1$. Тогда $\pi(A)=\{2, p\}, q-1$ является степенью 2, поэтому $q=2^{2^{n}}+1=p$. Отсюда $\mu(A)=\{p(p-1)\}, B$ - циклическая группа порядка $\left(p^{2}+1\right) / 2$, $C$ - циклическая группа порядка $(p+1) / 2$. Докажем, что группа $B$ простого порядка. Пусть это не так. Тогда $p_{1} p_{2}$ делит $|B|$, где $p_{1}, p_{2}$ - простые числа (возможно, одинаковые). Отсюда $|C|$ делит $p_{1}-1,|C|$ делит $p_{2}-1$, поэтому $p_{1}-1=|C| k_{1}=k_{1}(p+1) / 2$, $p_{2}-1=|C| k_{2}=k_{2}(p+1) / 2$, где $k_{1}, k_{2}$ - натуральные числа. Пусть $k_{i}=1$ для некоторого $i \in\{1,2\}$. Тогда $p_{i}=(p+3) / 2=2\left(2^{2^{n}-2}+1\right)-$ не простое число; противоречие. Итак, $k_{1}, k_{2} \geqslant 2$. Тогда

$$
\frac{p^{2}+1}{2}=|B| \geqslant p_{1} p_{2}=\left(\frac{p+1}{2} k_{1}+1\right)\left(\frac{p+1}{2} k_{2}+1\right) \geqslant p^{2} ;
$$

противоречие. Таким образом, $B$ простого порядка, т.е. $\left(p^{2}+1\right) / 2$ - простое число. По лемме 8 (б) имеем $p=3,5$, поэтому по условию $p=5$. Отсюда $\mu(A)=\{5 \cdot 4\}, B$ - циклическая группа порядка $13, C=\langle x\rangle$ - циклическая группа порядка 3 . По [5] $\mu(A C)=$ $\{12,20,30\}$. Из леммы $1(1)$ следует, что $A$ нильпотентна, поэтому $A=O_{2}(A) \times O_{5}(A)$. Так как в $A C$ есть элемент порядка 12 , существует элемент $y \in O_{2}(A)$ такой, что $|y|=4$, $x y=y x$. Так как в $A C$ есть элемент порядка 15 , существует элемент $z \in O_{5}(A)$ такой, что $|z|=5, x z=z x$. Тогда $|x y z|=60$, что противоречит строению $\mu(A C)$.

Теперь теорема следует из лемм 9-14.

\section{СПИСОК ЦИТИРОВАННОЙ ЛИТЕРАТУРЫ}

[1] Williams J.S. Prime graph components of finite groups // J. Algebra. 1981. V. 69. № 2. P. 487-513.

[2] Кондратьев А. С. О компонентах граффа простых чисел конечных простых групп // Матем. сб. 1989. Т. 180. №6. С. 787-797.

[3] Кондратьев А. С., Мазуров В. Д. Распознавание знакопеременных групп простой степени по порядкам их элементов // Сиб. матем. ж. 2000. Т. 41. № 2. С. 359-370.

[4] Aschbacher M. Finite Group Theory. Cambridge: Cambridge Univ. Press, 1986.

[5] Conway J. H., Curtis R. T., Norton S.P., Parker R. A., Wilson R. A. An Atlas of Finite Groups. Oxford: Clarendon Press, 1985.

[6] Thompson J. G. Normal p-complements for finite groups // Math. Z. 1960. V. 72. № 2 . P. 332-354.

[7] Zassenhaus H. Kennzeichnung endlicher linearean Gruppen als Permutationsgruppen // Abh. Math. Sem. Univ. Hamburg. 1936. V. 11. P. 17-40.

[8] Zassenhaus H. Über endliche Fastkörper // Abh. Math. Sem. Univ. Hamburg. 1936. V. 11. P. 187-220.

[9] Мазуров В. Д. Распознавание конечных простых групп $S_{4}(q)$ по их множеству порядков элементов // Алгебра и логика. 2002. Т. 41. С. 166-198.

[10] Мазуров В. Д., Су М. Ч., Чао Ч.П. Распознавание конечных простых групп $L_{3}\left(2^{m}\right)$ и $U_{3}\left(2^{m}\right)$ по порядкам их элементов // Алгебра и логика. 2000. Т. 39. № 5. С. 567-586.

[11] Мазуров В.Д. Характеризации конечных групп множествами порядков их элементов // Алгебра и логика. 1997. Т. 36. № 1. С. 36-53.

[12] Lipschutz S., Shi W. Finite groups whose element orders do not exceed twenty // Progress in Natural Sci. 2000. V. 10. № 1. P. 11-21.

[13] Shi W., Li H. L. A characteristic property of $M_{12}$ and $P S U(6,2) / /$ Acta Math. Sin. 1989. V. 32. № 6. P. 758-764.

[14] Shi W., Li H. L. A characteristic property of some sporadic simple groups // Chinese Ann. Math. Ser. A. 1993. V. 14. № 2. P. 144-151. 
[15] Shi W. The characterization of the sporadic simple groups by their element orders // Algebra Colloq. 1994. V. 1. № 2. P. 159-166.

[16] Заварницин А.В. Распознавание по множеству порядков элементов знакопеременных групп степени $r+1$ и $r+2$ для простого $r$ и группы степени $16 / /$ Алгебра и логика. 2000. T. 39. №6. C. $648-662$.

[17] Алеева М. Р. О композиционных факторах конечных групп с множеством порядков элементов как у группы $U_{3}(q) / /$ Сиб. матем. ж. 2002. Т. 43. № 2. С. 249-268.

[18] Белоногов В. А. Малые взаимодействия в группах $S L_{3}(q), S U_{3}(q), P S L_{3}(q)$ и $P S U_{3}(q)$ // Тр. ИММ УрО РАН. 1998. Т. 5. С. 3-27.

[19] Zsigmondy H. Zur Theorie der Potenzreste // Monatsh. Math. Phys. 1892. V. 3. P. 265-284.

[20] Семинар по алгебраическим группам. М.: Мир, 1975.

[21] Carter R. W. Conjugacy classes in the Weyl group // Compositio Math. 1972. V. 25. № 1. P. 1-59.

[22] Seitz G. M. On the subgroup structure of classical groups // Commun. Algebra. 1982. V. 10. № 8 . P. $875-885$.

[23] Deriziotis D. I. Conjugacy classes and centralizers of semisimple elements in finite groups of Lie type. Vorlesungen Fachb. Math. Univ. Essen., 1984. Heft 11.

[24] Srinivasan B. The characters of the finite symplectic group $S p(4, q) / /$ Trans. Amer. Math. Soc. 1968. V. 131. № 2. P. 488-525.

Институт математики и механики УрО РАН, г. Екатеринбург 